Article

\title{
Natural Convection Flow of Fractional Nanofluids Over an Isothermal Vertical Plate with Thermal Radiation
}

\author{
Constantin Fetecau ${ }^{1, *, \dagger}$, Dumitru Vieru ${ }^{2, \dagger}$ and Waqas Ali Azhar ${ }^{3,+}$ \\ 1 Academy of Romanian Scientists, Bucharest 050094, Romania \\ 2 Department of Theoretical Mechanics, Technical University of Iasi, Iasi 700050, Romania; \\ dumitru_vieru@yahoo.com \\ 3 Abdus Salam School of Mathematical Sciences, Government College University, Lahore 54000, Pakistan; \\ waqasaliazhar@gmail.com \\ * Correspondence: c_fetecau@yahoo.com; Tel.: +40-072-165-6339 \\ + These authors contributed equally to this work.
}

Academic Editor: Yulong Ding

Received: 27 November 2016; Accepted: 1 March 2017; Published: 3 March 2017

\begin{abstract}
The studies of classical nanofluids are restricted to models described by partial differential equations of integer order, and the memory effects are ignored. Fractional nanofluids, modeled by differential equations with Caputo time derivatives, are able to describe the influence of memory on the nanofluid behavior. In the present paper, heat and mass transfer characteristics of two water-based fractional nanofluids, containing nanoparticles of $\mathrm{CuO}$ and $\mathrm{Ag}$, over an infinite vertical plate with a uniform temperature and thermal radiation, are analytically and graphically studied. Closed form solutions are determined for the dimensionless temperature and velocity fields, and the corresponding Nusselt number and skin friction coefficient. These solutions, presented in equivalent forms in terms of the Wright function or its fractional derivatives, have also been reduced to the known solutions of ordinary nanofluids. The influence of the fractional parameter on the temperature, velocity, Nusselt number, and skin friction coefficient, is graphically underlined and discussed. The enhancement of heat transfer in the natural convection flows is lower for fractional nanofluids, in comparison to ordinary nanofluids. In both cases, the fluid temperature increases for increasing values of the nanoparticle volume fraction.
\end{abstract}

Keywords: free convection; thermal radiation; fractional nanofluids; exact solutions

\section{Introduction}

Natural convection flows have been extensively studied due to their multiple engineering applications. Such flows over an infinite plate are usually met in different engineering processes, including petroleum resource gas production, and geothermal reservoirs, thermal insulation, etc. (see [1-3]). The effects of thermal radiation are also important in geophysics, and geothermic, chemical, and ceramics processing, and they have been investigated by many researchers. A short presentation of the main results, up until 2007, is given by Ghosh and Beg [4], who studied the convective radiative heat transfer over a hot vertical surface in porous medium. Moreover, the effects of thermal radiation on nanofluid flows have been studied by many scholars. Mondal et al. [5] considered the unsteady magneto-hydrodynamic axi-symmetric stagnation-point flow over a shrinking sheet with Navier slip, and the temperature-dependent thermal conductivity. Magneto-hydrodynamic (MHD) flows of nanofluids, with radiation heat transfer over a flat plate with a variable heat flux and first-order chemical reaction, were studied by Zhang et al. [6]. A numerical study on Cu-water and Ag-water nanofluids, focusing on the radiation effects over a stretching sheet, was made by Abd Elazem et al. [7]. 
Nowadays, it is well known that one way of enhancing the thermal conductivity of fluids is by suspending the metallic particles, such as alumina, gold, copper, iron, or titanium, in fluids [8]. These particles, also called nanoparticles, have a diameter of less than $100 \mathrm{~nm}$ and the obtained solution is named nanofluid. The concept of a nanofluid seems to have been introduced by Choi [9], and based on his work, many researchers have focused their attention on the heat transfer in natural convection flows of nanofluids. Khan and Aziz [10] have studied the natural convection flow of a nanofluid over a vertical plate with a uniform surface heat flux. Turkyilmazoglu [11] provided exact solutions for the MHD slip flow of a nanofluid over a stretching/shrinking sheet, while Bachok et al. [12] studied the heat transfer characteristics on a moving plate in a nanofluid. Interesting exact solutions have also been obtained by Turkyilmazoglu and Pop [13], for the velocity and temperature fields corresponding to the natural convection flow of some nanofluids, past an infinite vertical plate with radiation effects. Radiation and magnetic effects on the natural convection flow of a nanofluid, past an infinite vertical plate with a heat source, have been studied by Mohankrishna et al. [14]. Ellahi [15] studied the effects of MHD and temperature-dependent viscosity on the flow of a non-Newtonian nanofluid in a pipe, while an analysis of the flow and heat transfer of water and ethylene glycol-based $\mathrm{Cu}$ nanoparticles between two parallel disks with suction/injection effects, has been provided by Rizwan Ul Haq et al. [16]. Of course, the list of such studies can continue, but we close it with some of the most interesting analytical and numerical results that have been obtained in [17-27].

However, none of these papers took into consideration the fractional derivatives in their governing equations, although the fractional models have been found to be quite flexible in describing the complex behavior of many materials. More recently, it seems that fractional partial differential equations may be used to describe some physical phenomena more accurately, when compared to the corresponding partial differential equations. Our interest here is to provide exact solutions for the temperature and velocity fields corresponding to the radiative natural convection flow of fractional nanofluids over an infinite vertical plate with heat and mass transfer, and to investigate the enhancement of heat transfer in such a flow, utilizing the fractional model. The associated skin friction coefficient and Nusselt number will be also determined. These solutions, which are presented in equivalent forms in terms of the Wright functions or its fractional derivatives, are reduced to similar solutions, corresponding to ordinary nanofluids [13]. Finally, the influence of the fractional parameter on the thermal and hydrodynamic response of physical interest, is graphically underlined and discussed.

\section{Statement of the Problem}

Let us consider the unsteady free convection flow and heat transfer of a nanofluid, modeled by the Caputo time-fractional derivative, past an infinite vertical plate situated in the $\left(x_{1}, z_{1}\right)$-plane of a fixed Cartesian coordinate system $O x_{1} y_{1} z_{1}$. At the initial moment $t_{1}=0$, the fluid and the plate are at rest, with a constant ambient temperature $T_{\infty}$. We also consider the radiation effect and assume the radiative heat flux to be applied, perpendicular to the plate. Since the plate is infinite, all of the physical quantities describing the fluid motion are functions of $y_{1}$ and $t_{1}$. The fluid is a water-based nanofluid containing nano particles of $\mathrm{CuO}$ or $\mathrm{Ag}$, whose thermo-physical properties are given in Table $1[13,28]$.

Table 1. Thermo-physical properties of water and nanoparticles.

\begin{tabular}{ccccc}
\hline Basic Fluid/Nanoparticles & $\boldsymbol{\rho}\left(\mathbf{K g} / \mathbf{m}^{\mathbf{3}}\right)$ & $C_{\boldsymbol{p}}(\mathbf{J} / \mathbf{K g} \mathbf{K})$ & $\boldsymbol{k}(\mathbf{W} / \mathbf{m} \cdot \mathbf{K})$ & $\boldsymbol{\beta} \times \mathbf{1 0 ^ { \mathbf { 5 } }} \mathbf{( \mathbf { 1 } / \mathbf { K } )}$ \\
\hline Pure water & 997.1 & 4179 & 0.613 & 21 \\
Copper oxide $(\mathrm{CuO})$ & 6320 & 531.8 & 76.5 & 1.80 \\
Silver $(\mathrm{Ag})$ & 10,500 & 235 & 429 & 1.89 \\
\hline
\end{tabular}


Assuming a small difference between the fluid temperature $T\left(y_{1}, t_{1}\right)$ and the stream temperature $T_{\infty}$, and adopting the Rosseland approximation [5], the radiative heat flux $q_{r}\left(y_{1}, t_{1}\right)$ can be linearized to:

$$
q_{r}\left(y_{1}, t_{1}\right)=-\frac{16 \sigma^{*} T_{\infty}^{3}}{3 k^{*}} \frac{\partial T\left(y_{1}, t_{1}\right)}{\partial y_{1}}
$$

where $\sigma^{*}$ is the Stefan-Boltzman constant and $k^{*}$ is the mean absorption coefficient.

In the following, we consider the nanofluid model proposed by Tiwari and Das [29], and take into consideration the usual Boussinesq's approximation. In this case, the governing equations can be written as [13]:

$$
\begin{gathered}
\rho_{n f} \frac{\partial u_{1}\left(y_{1}, t_{1}\right)}{\partial t_{1}}=\mu_{n f} \frac{\partial^{2} u_{1}\left(y_{1}, t_{1}\right)}{\partial y_{1}{ }^{2}}+g(\rho \beta)_{n f}\left[T\left(y_{1}, t_{1}\right)-T_{\infty}\right] ; y_{1}, t_{1}>0, \\
\left(\rho c_{p}\right)_{n f} \frac{\partial T\left(y_{1}, t_{1}\right)}{\partial t_{1}}=k_{n f}\left(1+\frac{16 \sigma^{*} T_{\infty}^{3}}{3 k_{n f} k^{*}}\right) \frac{\partial^{2} T\left(y_{1}, t_{1}\right)}{\partial y_{1}{ }^{2}} ; y_{1}, t_{1}>0 .
\end{gathered}
$$

If no slipping exists between the fluid and the plate, the appropriate initial and boundary conditions are:

$$
\begin{gathered}
u_{1}\left(y_{1}, 0\right)=0, T\left(y_{1}, 0\right)=T_{\infty} ; y_{1} \geq 0, \\
u_{1}\left(0, t_{1}\right)=0, T\left(0, t_{1}\right)=T_{w} ; t_{1}>0, \\
u_{1}\left(y_{1}, t_{1}\right) \rightarrow 0, T\left(y_{1}, t_{1}\right) \rightarrow 0 \text { as } y_{1} \rightarrow \infty .
\end{gathered}
$$

In the above relations, $u_{1}\left(y_{1}, t_{1}\right)$ is the fluid velocity in the $x_{1}$-vertical direction, $T_{w}$ is the constant plate temperature ( $T_{w}>T_{\infty}$ or $T_{w}<T_{\infty}$ corresponds to the heated or cooled plate, respectively), $g$ is the acceleration due to gravity, $\rho_{n f}$ is the density of the nanofluid, $\mu_{n f}$ is the dynamic viscosity of the nanofluid, and $\beta_{n f}$ is the thermal expansion coefficient of the nanofluid. Their expressions, as well as the expression of $\left(\rho c_{p}\right)_{n f}$, are given by:

$$
\begin{gathered}
\rho_{n f}=(1-\varphi) \rho_{f}+\varphi \rho_{s^{\prime}} \mu_{n f}=\frac{\mu_{f}}{(1-\varphi)^{2.5}} \\
(\rho \beta)_{n f}=(1-\varphi)(\rho \beta)_{f}+\varphi(\rho \beta)_{s^{\prime}},\left(\rho c_{p}\right)_{n f}=(1-\varphi)\left(\rho c_{p}\right)_{f}+\varphi\left(\rho c_{p}\right)_{s}
\end{gathered}
$$

where $\varphi$ is the nanoparticle volume fraction, $\rho_{f}$ is the density of the base fluid, $\rho_{s}$ is the density of the solid particle, and $c_{p}$ is the specific heat at constant pressure. The effective thermal conductivity of the nanofluid, corresponding to the Hamilton and Crosser model, is given by $[28,30]$ :

$$
\frac{k_{n f}}{k_{f}}=\frac{k_{s}+2 k_{f}-2 \varphi\left(k_{f}-k_{s}\right)}{k_{s}+2 k_{f}+\varphi\left(k_{f}-k_{s}\right)}
$$

where $k_{n f}, k_{f}$, and $k_{s}$ are the thermal conductivities of the nanofluid, the fluid, and the solid particles, respectively.

Next, the non dimensional variables and functions are introduced as:

$$
t=\frac{v_{f}}{L^{2}} t_{1}, y=\frac{y_{1}}{L}, u=\frac{L}{v_{f}} u_{1}, \theta=\frac{T-T_{\infty}}{T_{w}-T_{\infty}}, L=\left[\frac{v_{f}^{2}}{g \beta_{f}\left(T_{w}-T_{\infty}\right)}\right]^{1 / 3},
$$

Equations (2)-(6) take simplified dimensionless forms, as follows:

$$
\frac{\partial u(y, t)}{\partial t}=\frac{1}{a_{1}} \frac{\partial^{2} u(y, t)}{\partial y^{2}}+a_{2} \theta(y, t) ; y, t>0
$$




$$
\begin{gathered}
\frac{\partial \theta(y, t)}{\partial t}=\frac{1}{a_{3}} \frac{\partial^{2} \theta(y, t)}{\partial y^{2}} ; y, t>0, \\
u(y, 0)=0, \theta(y, 0)=0 ; y \geq 0, \\
u(0, t)=0, \theta(0, t)=1 ; t>0, \\
u(y, t) \rightarrow 0, \theta(y, t) \rightarrow 0 \text { as } y \rightarrow \infty,
\end{gathered}
$$

where:

$$
\begin{gathered}
a_{1}=(1-\varphi)^{2.5}\left[(1-\varphi)+\varphi \frac{\rho_{s}}{\rho_{f}}\right], \quad a_{2}=\frac{1-\varphi+\varphi \frac{(\rho \beta)_{s}}{(\rho \beta)_{f}}}{1-\varphi+\varphi \frac{\rho_{s}}{\rho_{f}}}, \\
a_{3}=\operatorname{Pr} \frac{1-\varphi+\varphi \frac{(\rho \rho p)_{s}}{(\rho c)_{f}}}{\frac{k_{n f}}{k_{f}}+N r}, \operatorname{Pr}=\frac{\mu_{f} c_{p f}}{k_{f}}, N r=\frac{16 \sigma^{*} T_{\omega^{3}}}{3 k^{*} k_{f}},
\end{gathered}
$$

where $P r$ is the Prandtl number and $N r$ is the radiation parameter.

The fractional model of the nanofluid is described by the fractional differential equations:

$$
\begin{gathered}
{ }^{c} D_{t}^{\alpha} u(y, t)=\frac{1}{a_{1}} \frac{\partial^{2} u(y, t)}{\partial y^{2}}+a_{2} \theta(y, t) ; y, t>0, \\
{ }^{c} D_{t}^{\alpha} \theta(y, t)=\frac{1}{a_{3}} \frac{\partial^{2} \theta(y, t)}{\partial y^{2}} ; y, t>0,
\end{gathered}
$$

together with the initial and boundary conditions given by Equations (13)-(15). The operator ${ }^{c} D_{t}^{\alpha}$ represents the Caputo time-fractional derivative, defined as [31]:

$$
{ }^{c} D_{t}^{\alpha} u(y, t)=\frac{1}{\Gamma(1-\alpha)} \int_{0}^{t}(t-s)^{-\alpha} \frac{\partial u(y, s)}{\partial s} d s ; 0 \leq \alpha<1 .
$$

The Caputo derivative is:

$$
L\left\{{ }^{c} D_{t}^{\alpha} u(y, t)\right\}(q)=q^{\alpha} \bar{u}(y, q)-q^{\alpha-1} u(y, 0) \text { if } \bar{u}(y, q)=L\{u(y, t)\}(q)
$$

and:

$$
\lim _{\alpha \rightarrow 1}^{c} D_{t}^{\alpha} u(y, t)=\frac{\partial u(y, t)}{\partial t}
$$

\section{Solution of the Problem}

In order to determine the solution of the fractional partial differential Equations (17) and (18), with the initial and boundary conditions (13)-(15), the Laplace transform technique will be used. Equation (18) is not coupled to the momentum equation. Consequently, we shall firstly determine the temperature field.

\subsection{Determination of the Temperature Field}

Applying the Laplace transform to Equation (18), and bearing in mind the corresponding initial and boundary conditions, we find that:

$$
a_{3} q^{\alpha} \bar{\theta}(y, q)=\frac{\partial^{2} \bar{\theta}(y, q)}{\partial y^{2}} ; y>0
$$


where $q$ is the transform parameter and the Laplace transform $\bar{\theta}(y, q)$ of $\theta(y, t)$ has to satisfy the following conditions:

$$
\bar{\theta}(0, q)=\frac{1}{q} ; \bar{\theta}(y, q) \rightarrow 0 \text { as } y \rightarrow \infty
$$

The solution to the problems (22) and (23) is:

$$
\bar{\theta}(y, q)=\frac{1}{q} \exp \left(-y \sqrt{a_{3} q^{\alpha}}\right) ; y>0 .
$$

Applying the inverse Laplace transform to Equation (24), and using Equation (A1) from the Appendix A, we find that:

$$
\theta(y, t)=\Psi\left(1, \frac{-\alpha}{2} ;-y \sqrt{a_{3}} t^{-\frac{\alpha}{2}}\right) \text { for } 0<\alpha \leq 1,
$$

where:

$$
\Psi(a,-b ; z)=\sum_{n=1}^{\infty} \frac{z^{n}}{n ! \Gamma(a-n b)} ; b \in(0,1),
$$

is the Wright function [32]. For $\alpha=1$, Equation (25) becomes:

$$
\theta(y, t)=\operatorname{erfc}\left(\frac{y \sqrt{a_{3}}}{2 \sqrt{t}}\right) ; y, t>0 .
$$

Of course, a simple analysis clearly shows that this result is in accordance with that obtained by Turkyilmazoglu and Pop [13], Equation (3.19).

\subsection{Calculation of the Velocity Field}

Applying the Laplace transform to Equation (17), and taking into consideration the corresponding initial and boundary conditions, we find that:

$$
\frac{\partial^{2} \bar{u}(y, q)}{\partial y^{2}}-a_{1} q^{\alpha} \bar{u}(y, q)=-a_{1} a_{2} \frac{1}{q} \exp \left(-y \sqrt{a_{3} q^{\alpha}}\right) ; y>0
$$

where the Laplace transform $\bar{u}(y, q)$ of $u(y, t)$ has to satisfy the conditions:

$$
\bar{u}(0, q)=0 ; \bar{u}(y, q) \rightarrow 0 \text { as } y \rightarrow \infty .
$$

A particular solution of Equation (28) is:

$$
\bar{u}_{p}(y, q)=\frac{a_{1} a_{2}}{a_{1}-a_{3}} \frac{1}{q^{\alpha+1}} \exp \left(-y \sqrt{a_{3} q^{\alpha}}\right),
$$

while its general solution is:

$$
\bar{u}(y, q)=A \exp \left(y \sqrt{a_{1} q^{\alpha}}\right)+B \exp \left(-y \sqrt{a_{1} q^{\alpha}}\right)+\frac{a_{1} a_{2}}{a_{1}-a_{3}} \frac{1}{q^{\alpha+1}} \exp \left(-y \sqrt{a_{3} q^{\alpha}}\right) .
$$

Considering the conditions of (29), it results that:

$$
\bar{u}(y, q)=\frac{a_{1} a_{2}}{a_{1}-a_{3}} \frac{1}{q^{\alpha+1}}\left[\exp \left(-y \sqrt{a_{3} q^{\alpha}}\right)-\exp \left(-y \sqrt{a_{1} q^{\alpha}}\right)\right] ; 0<\alpha<1 .
$$


Applying the inverse Laplace transform to Equation (32), using the convolution theorem and the Equality (A1), we find that:

$$
u(y, t)=\frac{a_{1} a_{2}}{a_{1}-a_{3}} \frac{1}{\Gamma(1-\beta)} \int_{0}^{t} \frac{1}{(t-s)^{\beta}}\left[\Psi\left(1, \frac{\beta-1}{2} ;-y \sqrt{a_{3} s^{\beta-1}}\right)-\Psi\left(1, \frac{\beta-1}{2} ;-y \sqrt{a_{1} s^{\beta-1}}\right)\right] d s,
$$

where $\beta=1-\alpha$.

Now, using the identitiy (A2) from the Appendix A, we can present our solution in an interesting, but equivalent, form:

$$
u(y, t)=\frac{a_{1} a_{2}}{a_{1}-a_{3}}\left\{{ }^{c} D_{t}^{\beta}\left[t \Psi\left(2, \frac{\beta-1}{2} ;-y \sqrt{a_{3} t^{\beta-1}}\right)\right]-{ }^{c} D_{t}^{\beta}\left[t \Psi\left(2, \frac{\beta-1}{2} ;-y \sqrt{a_{1} t^{\beta-1}}\right)\right]\right\},
$$

in terms of the Caputo derivative of the Wright functions.

\subsection{Nusselt Number and Skin Friction}

In order to determine the two entities of physical interest, namely the Nusselt number $N u$ and the skin friction coefficient $C_{f}$, we use the relations:

$$
\begin{gathered}
N u=\frac{L q_{w}}{k_{f}\left(T_{w}-T_{\infty}\right)}=-\left.\frac{L k_{n f}}{k_{f}\left(T_{w w}-T_{\infty}\right)} \frac{\partial T\left(y_{1}, t_{1}\right)}{\partial y_{1}}\right|_{y_{1}=0}= \\
-\left.\frac{k_{n f}}{k_{f}} \frac{\partial \theta(y, t)}{\partial y}\right|_{y=0}=-\frac{k_{n f}}{k_{f}} \lim _{y \rightarrow 0^{+}} L^{-1}\left\{\frac{\partial \bar{\theta}(y, q)}{\partial y}\right\} . \\
C_{f}=\frac{\tau_{w}}{\rho_{f}\left(\frac{v_{f}}{L}\right)^{2}}=\left.\frac{\mu_{n f}}{\rho_{f}\left(\frac{v_{f}}{L}\right)^{2}} \frac{\partial u_{1}\left(y_{1}, t_{1}\right)}{\partial y_{1}}\right|_{y_{1}=0}= \\
\left.\frac{\mu_{n f}}{\mu_{f}} \frac{\partial u(y, t)}{\partial y}\right|_{y=0}=\frac{1}{(1-\varphi)^{2.5}} \lim _{y \rightarrow 0^{+}} L^{-1}\left\{\frac{\partial \bar{u}(y, q)}{\partial y}\right\},
\end{gathered}
$$

where $q_{w}$ is the constant heat flux from the surface of the plate and $\tau_{w}$ is the skin friction or shear stress on the boundary.

Introducing Equations (24) and (32) into (35) and (36), respectively, we find that:

$$
N u=\frac{k_{n f}}{k_{f}} \sqrt{a_{3}} \frac{t^{-\frac{\alpha}{2}}}{\Gamma\left(1-\frac{\alpha}{2}\right)}, \quad C_{f}=\frac{a_{1} a_{2}}{\sqrt{a_{1}}+\sqrt{a_{2}}} \frac{t^{\frac{\alpha}{2}}}{\Gamma\left(1+\frac{\alpha}{2}\right)} \frac{1}{(1-\varphi)^{2.5}} .
$$

Using the identity (A3) from the Appendix A, we also provide equivalent forms for $\mathrm{Nu}$ and $C_{f}$, namely:

$$
N u=2 \frac{k_{n f}}{k_{f}} \sqrt{{\frac{a_{3}}{\pi}}^{c}} D_{t}^{\frac{1+\alpha}{2}}\left(t^{1 / 2}\right), \quad C_{f}=\frac{1}{(1-\varphi)^{2.5}} \frac{a_{1} a_{2}}{\sqrt{a_{1}}+{\sqrt{a_{2}}}^{c}} D_{t}^{1-\frac{\alpha}{2}}(t)
$$

in terms of the Caputo derivatives of $t^{1 / 2}$ and $t$.

\section{Validation}

In order to bring to light the accuracy of the results that have been obtained, it is suitable to show that they are in accordance with similar solutions from the existing literature. For that, let us use $\beta=0$ in Equation (34). It corresponds to $\alpha=1$, and the solution corresponding to the same unsteady natural convection flow of ordinary nanofluids, has to be obtained. When the Caputo derivative of zero order is the identity operator, Equation (34) becomes:

$$
u(y, t)=\frac{a_{1} a_{2}}{a_{1}-a_{3}}\left\{\left[t \Psi\left(2, \frac{-1}{2} ;-y \sqrt{\frac{a_{3}}{t}}\right)\right]-\left[t \Psi\left(2, \frac{-1}{2} ;-y \sqrt{\frac{a_{1}}{t}}\right)\right]\right\} .
$$


On the other hand (see also Equation (A2)):

$$
\operatorname{erfc}\left(\frac{y \sqrt{a}}{2 \sqrt{t}}\right)=\Psi\left(1, \frac{-1}{2} ;-y \sqrt{\frac{a}{t}}\right)=\frac{\partial}{\partial t}\left[t \Psi\left(2, \frac{-1}{2} ;-y \sqrt{\frac{a}{t}}\right)\right]
$$

and then:

$$
t \Psi\left(2, \frac{-1}{2} ;-y \sqrt{\frac{a}{t}}\right)=\int_{0}^{t} \operatorname{erfc}\left(\frac{y \sqrt{a}}{2 \sqrt{s}}\right) d s,
$$

where $\operatorname{erf} c(\cdot)$ is the complementary error fucntion of Gauss.

Now, by introducing Equation (41) in (39), and by using Equation (A4), we get the velocity field as the simple form:

$$
u(y, t)=\frac{a_{1} a_{2}}{a_{1}-a_{3}}\left\{\begin{array}{l}
\left(t+\frac{y^{2} a_{3}}{2}\right) \operatorname{erfc}\left(\frac{y \sqrt{a_{3}}}{2 \sqrt{t}}\right)-\left(t+\frac{y^{2} a_{1}}{2}\right) \operatorname{erfc}\left(\frac{y \sqrt{a_{1}}}{2 \sqrt{t}}\right)+ \\
\frac{y \sqrt{a_{1} t}}{\sqrt{\pi}} \exp \left(-\frac{y^{2} a_{1}}{4 t}\right)-\frac{y \sqrt{a_{3} t}}{\sqrt{\pi}} \exp \left(-\frac{y^{2} a_{3}}{4 t}\right)
\end{array}\right\}
$$

Finally, bearing in mind the notations of Turkyilmazoglu and Pop [13], as well as their rescaling relation from equality (2.11), it is easy to show that our solution (42) is identical to Equation (3.20), from [13].

With regards to the Nusselt number $N u$ and the skin friction coefficient $C_{f}$, we use $\alpha=1$ in Equation (38) and use Equation (A5). The expressions corresponding to the ordinary nanofluid are:

$$
N u=\frac{k_{n f}}{k_{f}} \frac{\sqrt{a_{3}}}{\sqrt{\pi t}}, \quad C_{f}=\frac{1}{(1-\varphi)^{2.5}} \frac{2 a_{1} a_{2}}{\sqrt{a_{1}}+\sqrt{a_{3}}} \sqrt{\frac{t}{\pi}}
$$

As expected, by changing $a_{1}, a_{3}$, and $t$, by $\frac{1}{a_{1}}, \frac{1}{a_{3}}$, and $\tau_{1}$, respectively, we recover the solutions (3.21), from [13].

\section{Numerical Results and Discussion}

The natural convection flow of water-based fractional nanofluids over an infinite vertical plate with thermal radiation and a uniform temperature on the boundary, is analytically studied. Closed form solutions for the dimensionless temperature and velocity fields, and the two entities of physical interest, the Nusselt number and skin friction coefficient, are determined in equivalent forms, in terms of the Wright function or its fractional derivatives. It is worth pointing out that all of these solutions have been immediately reduced to the known solutions, based on the literature for ordinary nanofluids. A table containing the thermo-physical properties of copper oxide $(\mathrm{CuO})$ and silver $(\mathrm{Ag})$ is also included for later use.

In order to bring to light the influence of the fractional parameter on the heat and mass transfer in the natural convection flow of the above-mentioned fractional nanofluids, and therefore to obtain some physical insight into the present results, some numerical calculations have been carried out for different values of the fractional parameter $\alpha$, radiation parameter $N r$, and the nanoparticle value fraction $\varphi$. For comparison, the diagrams of dimensionless temperature and velocity fields, and the Nusselt number and skin friction coefficient corresponding to fractional nanofluids (for different values of the fractional parameter $\alpha \in(0,1)$ ) and those of ordinary nanofluids (when $\alpha=1$ ), are depicted in Figures 1-4. As was expected, in all cases, the diagrams corresponding to the fractional nanofluids tend to superpose over those of ordinary nanofluids, when $\alpha \rightarrow 1$.

Profiles of the dimensionless temperature $\theta(y, t)$ against y are presented in Figure $1 \mathrm{a}-\mathrm{c}$, for the different values of the fractional parameter $\alpha$ and the $\mathrm{CuO}$ nanoparticle volume fraction $\varphi$. The fluid temperature, as it results from these figures, increases with respect to $\alpha$, up to a critical value of $y$ (less than 0.5), and then decreases. Consequently, in terms of the plate proximity, the heat transfer is stronger when the thermal boundary layer is thinner for fractional nanofluids, in comparison to 
the ordinary ones. An opposite trend appears at a later point in time. With respect to the volume fraction $\varphi$, the temperature is an increasing function for both ordinary and fractional nanofluids, and it smoothly decreases from the maximum value of one on the boundary, to the zero value far away from the plate. In Table 2, the temperature at different values of $y$ and of the fractional parameter $\alpha$, is given for nanofluids containing nanoparticles of $\mathrm{CuO}$ or $\mathrm{Ag}$, and these values are in full accordance with those resulting from Figure 1. Furthermore, as results from this table, the Ag nanoparticles induce larger temperature values and a lower heat transfer to ordinary or fractional nanofluids, in comparison to $\mathrm{CuO}$ nanoparticles.

The effect of enhancing the heat transfer rate against $\varphi$ with the fractional parameter $\alpha$, for $\mathrm{CuO}$-water and Ag-water nanofluids, is presented in Figure 2, in the absence or presence of thermal radiation. For both fractional nanofluids, the Nusselt number $\mathrm{Nu}$ is an increasing function with respect to $\alpha$. It is also an almost linearly increasing function of $\varphi$. Further, the heat transfer rate for the $\mathrm{CuO}$-water fractional nanofluid is always a little higher than that corresponding to Ag-water fractional nanofluid. However, the difference between them increases with increasing values of $\varphi$.

The influence of the fractional parameter $\alpha$ and of the nanoparticle volume fraction $\varphi$, on the dimensionless velocity $u(y, t)$ against $y$, is brought to light by Figure $3 a-c$; which is also seen for a nanofluid with $\mathrm{CuO}$ nanoparticles. Near the plate, the nanofluid velocity increases up to a maximum value and then asymptotically decreases to the zero value for $y$ values greater than 2.5 , but it is a decreasing function with respect to the two parameters $\alpha$ and $\varphi$. Consequently, the boundary layer thickness is lower for ordinary nanofields, in comparison to fractional nanofluids. From a physical point of view, it means that the nanofluid viscosity decreases for increasing values of $\alpha$ or $\varphi$, and the ordinary nanofluids exhibit a stronger capacity in flow. This implies that the viscoelasticity strengthens the flow resistance with a decrease in the fractional parameter [33]. The variation of the skin friction coefficient $C_{f}$, against the nanoparticle volume fraction $\varphi$, is presented in Figure 4, for the same nanofluids at different values of $\alpha$ and $N r$, equal to zero or one.

Table 2. Values of the dimensionless temperature for $\varphi=0.2, t=0.5$, and different values of the fractional parameters $\alpha$ and $y$.

\begin{tabular}{|c|c|c|c|c|c|c|c|c|}
\hline \multirow{2}{*}{$y$} & \multicolumn{4}{|c|}{$\mathrm{CuO}$} & \multicolumn{4}{|c|}{ Ag } \\
\hline & $\alpha=0.1$ & $\alpha=0.5$ & $\alpha=0.85$ & $\alpha=1$ & $\alpha=0.1$ & $\alpha=0.5$ & $\alpha=0.85$ & $\alpha=1$ \\
\hline 0 & 1 & 1 & 1 & 1 & 1 & 1 & 1 & 1 \\
\hline 0.1 & 0.86341 & 0.84348 & 0.84816 & 0.87689 & 0.86675 & 0.84694 & 0.85013 & 0.87864 \\
\hline 0.2 & 0.74363 & 0.72536 & 0.75925 & 0.77086 & 0.74937 & 0.73083 & 0.76448 & 0.7768 \\
\hline 0.3 & 0.64057 & 0.62803 & 0.65475 & 0.6609 & 0.648 & 0.63504 & 0.66273 & 0.6691 \\
\hline 0.4 & 0.55187 & 0.54341 & 0.55664 & 0.55868 & 0.56041 & 0.55166 & 0.56628 & 0.56886 \\
\hline 0.5 & 0.47551 & 0.46851 & 0.46887 & 0.46476 & 0.48472 & 0.47766 & 0.47961 & 0.47635 \\
\hline 0.6 & 0.40976 & 0.40218 & 0.39111 & 0.38035 & 0.4193 & 0.4119 & 0.4025 & 0.39275 \\
\hline 0.7 & 0.35313 & 0.34379 & 0.32302 & 0.30608 & 0.36274 & 0.35376 & 0.33461 & 0.31872 \\
\hline 0.8 & 0.30436 & 0.29277 & 0.26415 & 0.24213 & 0.31383 & 0.30272 & 0.27556 & 0.25448 \\
\hline 0.9 & 0.26234 & 0.2485 & 0.21388 & 0.18821 & 0.27154 & 0.25821 & 0.22481 & 0.19985 \\
\hline 1 & 0.22613 & 0.2103 & 0.17148 & 0.14371 & 0.23496 & 0.2196 & 0.1817 & 0.15432 \\
\hline
\end{tabular}

The variation of the skin friction coefficient $C_{f}$, against the nanoparticle volume fraction $\varphi$, is presented in Figure 4, for the same nanofluids at different values of $\alpha$ and $N r$, equal to zero or one. In both cases, i.e., in the absence or presence of radiation, the skin friction coefficient is a decreasing function with respect to the fractional parameter $\alpha$. Considering its variation with respect to $\varphi$, two different situations appear. In the absence of radiation (Figure $4 \mathrm{~b}$ ), it is an increasing function with respect to $\varphi$, and its values are always greater for Ag-water fractional nanofluid compared to $\mathrm{CuO}$-water fractional nanofluid. In the presence of radiation, when $\mathrm{Nr}=1$ (Figure 4a), the shear stress on the plate decreases up to a critical value of $\varphi$ (about 0.1), and then increases. Furthermore, up to this value of $\varphi$, it is smaller for Ag-water fractional nanofluid in comparison to CuO-water fractional 
nanofluid, and a reverse situation is presented at a later point in time. Throughout this study, the value of $\operatorname{Pr}$ has been taken as 6.067 .
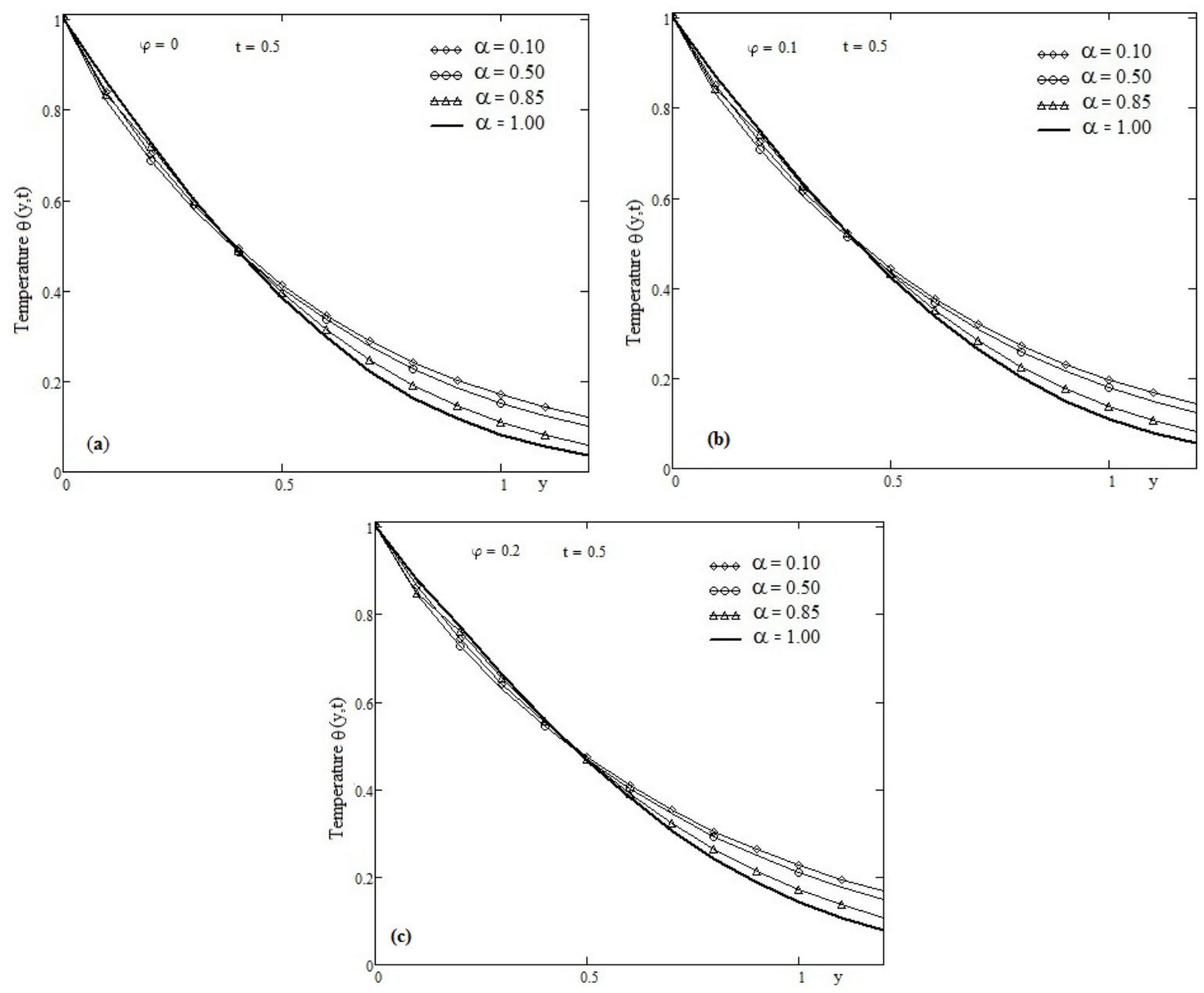

Figure 1. Variation of the dimensionless temperature $\theta(y, t)$ with the fractional parameter $\alpha$ and the $\mathrm{CuO}$ nanoparticle volume fraction $\varphi$. (a) for $\varphi=0$; (b) for $\varphi=0.1$; (c) for $\varphi=0.2$.

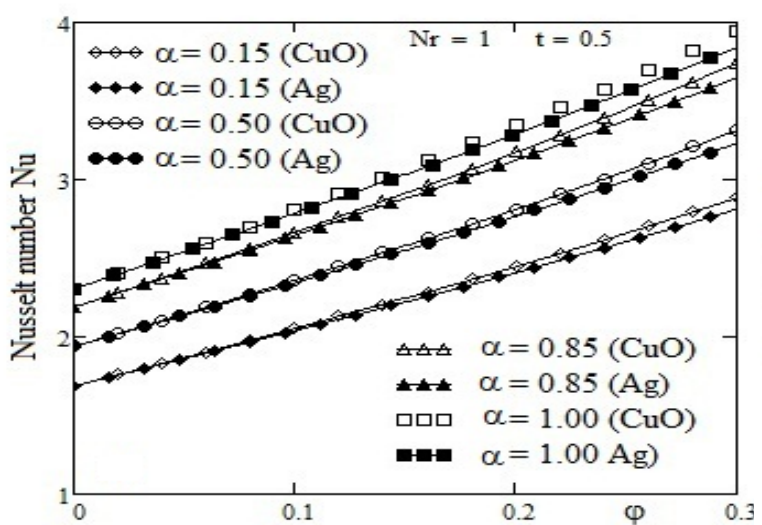

(a)

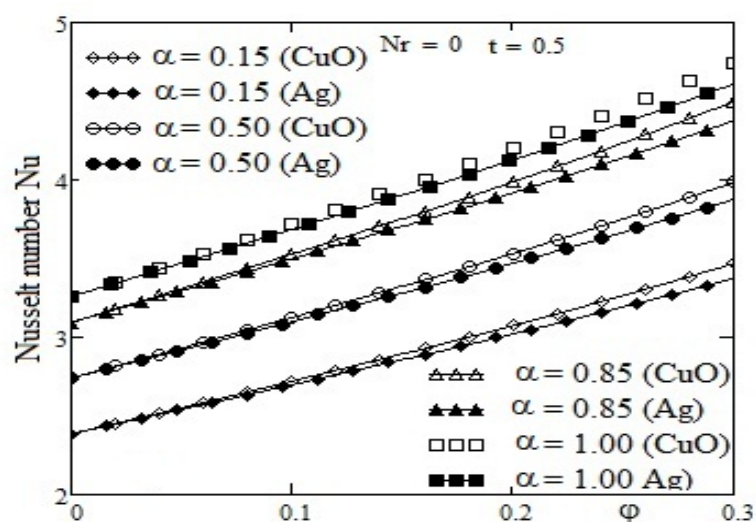

(b)

Figure 2. Values of the Nusselt number against the nanoparticle volume fraction $\varphi$ for the two water-based nanofluids. (a) for $N r=1$; (b) for $N r=0$. 

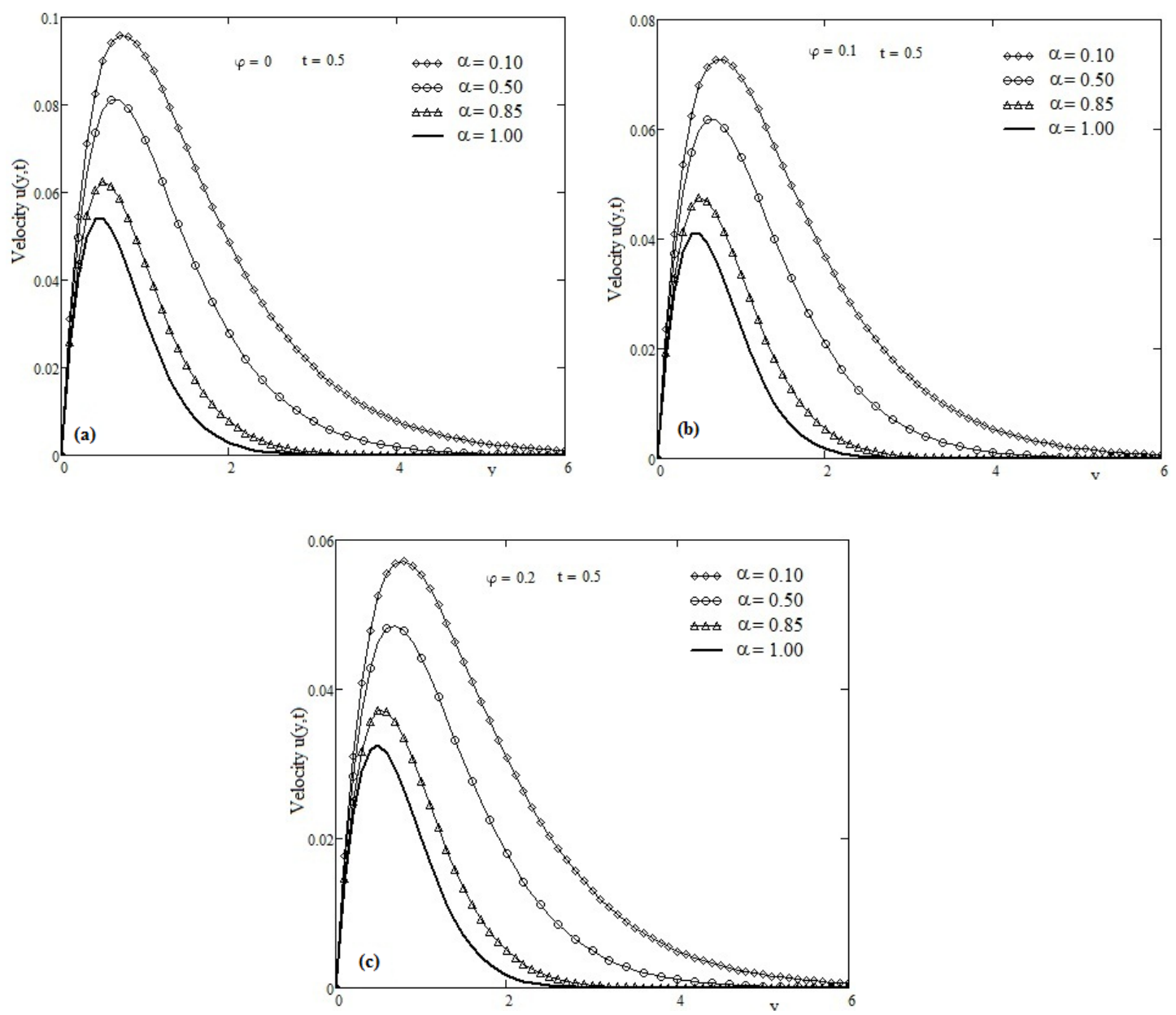

Figure 3. Variation of the velocity $u(y, t)$ with the fractional parameter $\alpha$ and the nanoparticle volume fraction $\varphi$. (a) for $\varphi=0$; (b) $\varphi=0.1$; (c) for $\varphi=0.2$.

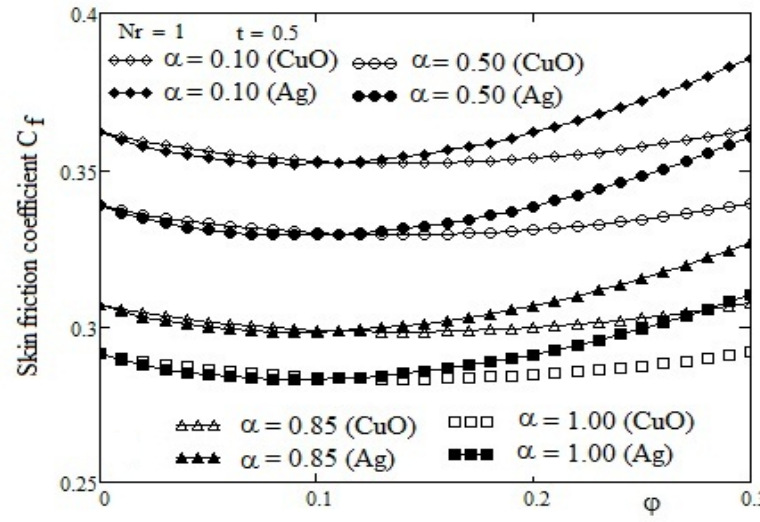

(a)

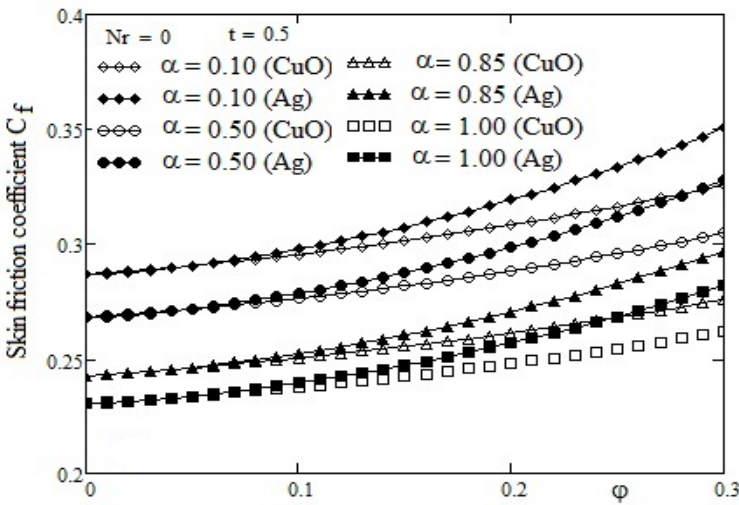

(b)

Figure 4. Values of the skin friction coefficient $C_{f}$ against the nanoparticle volume fraction $\varphi$ for two water-based fractional nanofluids. (a) for $N r=1$; (b) for $N r=0$.

\section{Conclusions}

An analytical study of the natural convection flow of some water-based fractional nanofluids over an infinite vertical plate with thermal radiation and a uniform temperature on the boundary, 
is developed using the Caputo time-fractional derivative. The closed forms of solutions for the dimensionless temperature and velocity fields, and the corresponding Nusselt number and skin friction coefficient, are established in equivalent forms, in terms of the Wright function and its fractional derivatives. They have been reduced to the solutions obtained in [13], corresponding to ordinary nanofluids, when the fractional parameter $\alpha \rightarrow 1$.

In order to get some physical insight into the results, which have been obtained for $\mathrm{CuO}$-water and Ag-water fractional nanofluids, some numerical calculations and graphical representations have been presented in Table 2 and Figures 1-4, for different values of the fractional parameter $\alpha$, radiative parameter $N r$, and the nanoparticles volume fraction $\varphi$. The main findings are:

The enhancement of heat transfer in the natural convection flows is lower for fractional nanofluids, in comparison to ordinary nanofluids. The thermal boundary layer is thicker for fractional nanofluids. In both cases, the fluid temperature increases and the heat transfer declines for increasing values of the nanoparticle volume fraction $\varphi$.

The flows of water-based fractional nanofluids are faster than the ordinary nanofluids.

A decrease in the fractional derivative parameter increases the thickness of the velocity boundary layer. From a physical point of view, it means that the nanofluid viscosity increases with the decreasing of $\alpha$.

The dimensionless velocity of water-based fractional nanofluids, as well as that of ordinary nanofluids, is a decreasing function with respect to the nanoparticle volume fraction $\varphi$.

The skin friction coefficient in the natural convection flow is higher for fractional nanofluids, in comparison to ordinary nanofluids.

The enhancement of heat transfer in natural convection is stronger for $\mathrm{Cu}$-water fractional nanofluids, when compared with Ag-water fractional nanofluids.

In the presence of radiation, Ag-water fractional/ordinary nanofluids achieve a lower skin friction coefficient near the plate, relative to $\mathrm{Cu}$-water fractional/ordinary nanofluids.

Our study shows that the nanofluids described by fractional derivatives have a significantly different behavior than ordinary nanofluids. The same conclusions were obtained in the references [33,34]. Even if the models studied in these articles are different from the model studied here, the obtained results showed that the fractional parameter has a strong influence on the heat transfer process.

Acknowledgments: Waqas Ali Azhar is grateful to Abdus Salam School of Mathematical Sciences, Government College University, Lahore, Pakistan for supporting this scientific research.

Conflicts of Interest: The authors declare no conflict of interest.

\section{Appendix}

$$
\begin{gathered}
L^{-1}\left\{\frac{1}{q^{a}} \exp \left(-c q^{b}\right)\right\}=t^{a-1} \Psi\left(a,-b ;-c t^{-b}\right) ; \quad b \in(0,1) \\
\frac{\partial}{\partial t}\left[t \Psi\left(2,-b ;-c t^{-b}\right)\right]=\Psi\left(1,-b ;-c t^{-b}\right) ; b \in(0,1) \\
{ }^{c} D_{t}^{\alpha}\left(t^{\gamma}\right)=\frac{\Gamma(\gamma+1)}{\Gamma(\gamma+1-\alpha)} t^{\gamma-\alpha} \\
\int_{0}^{t} \operatorname{erfc}\left(\frac{y \sqrt{a}}{2 \sqrt{s}}\right) d s=\left(t+\frac{a y^{2}}{2}\right) \operatorname{erfc}\left(\frac{y \sqrt{a}}{2 t}\right)-\frac{y \sqrt{a}}{\sqrt{\pi}} \exp \left(\frac{-a y^{2}}{4 t}\right) \\
{ }^{c} D_{t}^{1}\left(t^{1 / 2}\right)=\frac{1}{2 \sqrt{t}},{ }^{c} D_{t}^{1 / 2}(t)=\frac{2}{\sqrt{\pi}} \sqrt{t}
\end{gathered}
$$




\section{References}

1. Raptis, A. Unsteady free convection flow through a porous medium. Int. J. Eng. Sci. 1983, 21, 345-348. [CrossRef]

2. Kim, S.J.; Vafai, K. Analysis of natural convection about a vertical plate embedded in a porous medium. Int. J. Heat Mass Transfer 1989, 32, 665-677. [CrossRef]

3. Narasimha, K.R.; Pop, I. Transient free convection in a fluid saturated porous media with temperature dependent viscosity. Int. Commun. Heat Mass Transf. 1994, 21, 573-581.

4. Ghosh, S.K.; Beg, O.A. Theoretical analysis of radiative effects on transient free convection heat transfer past a hot vertical surface in porous media. Nonlinear Anal. Model. Control 2008, 13, 419-432.

5. Mondal, S.; Haroun, N.A.H.; Sibanda, P. The effects of thermal radiation on an unsteady MHD axisymmetric stagnation-point flow over a shrinking sheet in presence of temperature dependent thermal conductivity with Navier slip. PLoS ONE 2015, 10, e0138355. [CrossRef] [PubMed]

6. Zhang, C.; Zheng, L.; Zhang, X.; Chen, G. MHD flow and radiation heat transfer of nanofluids in porous media with variable surface heat flux and chemical reaction. Appl. Math. Model. 2015, 39, 165-181. [CrossRef]

7. Abd Elazem, N.Y.; Ebaid, A.; Aly, H.E. Radiation effect of MHD on Cu-water and Ag-water nanofluids flow over a stretching sheet: Numerical study. J. Appl. Computat. Math. 2015, 4. [CrossRef]

8. Bianco, V.; Manca, O.; Nardini, S.; Vafai, K. Heat Transfer Enhancement with Nanofluids; CRC Press: London, UK, 2015.

9. Choi, S.U.S. Enhancing thermal conductivity of fluids with nanoparticles. In Proceedings of the ASME International Mechanical Engineering Congress and Exposition 66, San Francisco, CA, USA, 12-17 November 1995; pp. 99-105.

10. Khan, W.A.; Aziz, A. Natural convection flow of a nanofluid over a vertical plate with uniform surface heat flux. Int. J. Therm. Sci. 2011, 50, 1207-1214. [CrossRef]

11. Turkyilmazoglu, M. Exact analytical solutions for heat and mass transfer of MHD slip flow in nanofluids. Chem. Eng. Sci. 2012, 84, 182-187. [CrossRef]

12. Bachok, N.; Ishak, A.; Pop, I. Flow and heat transfer characteristics on a moving plate in a nanofluid. Int. J. Heat Mass Transf. 2012, 55, 642-648. [CrossRef]

13. Turkyilmazoglu, M.; Pop, I. Heat and mass transfer of unsteady natural convection flow of some nanofluids past a vertical infinite flat plate with radiation effect. Int. J. Heat Mass Transf. 2013, 59, 167-171. [CrossRef]

14. Mohankrishna, P.; Sugunamma, V.; Sandeep, N. Radiation and magnetic field effects on unsteady natural convection flow of a nanofluid past an infinite vertical plate with heat source. Chem. Process. Eng. Res. 2014, 25, 39-52.

15. Ellahi, R. The effects of MHD and temperature dependent viscosity on the flow of non-Newtonian nanofluid in a pipe: Analytical solutions. Appl. Math. Model. 2013, 37, 1451-1457. [CrossRef]

16. Rizwan, U.H.; Khan, Z.H.; Hussain, S.T.; Hammouch, Z. Flow and heat transfer analysis of water and ethylene glycol based $\mathrm{Cu}$ nanoparticles between two parallel disks with suction/injection effects. J. Mol. Liq. 2016, 221, 298-304. [CrossRef]

17. Sheikholeslami, M.; Zaigham, Q.M.; Ellahi, R. Influence of induced magnetic field on free convection of nanofluid considering Koo-Kleinstreuer-Li (KKL) correlation. Appl. Sci. 2016, 324, 1-13. [CrossRef]

18. Sekrani, G.; Poncet, S. Further investigation on laminar forced convection of nanofluid flows in a uniformly heated pipe using direct numerical simulations. Appl. Sci. 2016, 332, 1-24. [CrossRef]

19. Rizwan, U.H.; Prabhakar, B.; Bandari, S. Impact of inclined Lorentz forces on Tangent hyperbolic nanofluid flow with zero normal flux of nanoparticles at the stretching sheet. Neural Comput. Appl. 2016. [CrossRef]

20. Ibrahim, W.; Ul Haq, R. Magnetohydrodynamic (MHD) stagnation point flow of nanofluid past a stretching sheet with convective boundary condition. J. Braz. Soc. Mech. Sci. Eng. 2016, 38, 1155-1164. [CrossRef]

21. Waqar, A.; Khan, C.R.; Rizwan, U.H. Heat Transfer Analysis of MHD Water Functionalized Carbon Nanotube Flow over a Static/Moving Wedge. J. Nanomater. 2015, 16, 934367. [CrossRef]

22. Khan, W.A.; Khan, Z.H.; Haq, R.U. Flow and heat transfer of ferrofluids over a flat plate with uniform heat flux. Eur. Phys. J. Plus 2015, 130. [CrossRef]

23. Rizwan, U.H.; Rajotia, D.; Noor, N.F.M. Thermophysical effects of water driven copper nanoparticles on MHD axisymmetric permeable shrinking sheet: Dual-nature study. Eur. Phys. J. 2016, 39. [CrossRef] 
24. Sheikholeslami, M.; Ellahi, R. Electrohydrodynamic nanofluid hydrothermal treatment in an enclosure with sinusoidal upper wall. Appl. Sci. 2015, 5, 294-306. [CrossRef]

25. Rahman, S.U.; Ellahi, R.; Nadeem, S.; Zia, Q.M. Simultaneous effects of nanoparticles and slip on Jeffrey fluid through tapered artery with mild stenosis. J. Mol. Liq. 2016, 218, 484-493. [CrossRef]

26. Sheikholeslami, M.; Ellahi, R. Three dimensional mesoscopic simulation of magnetic field effect on natural convection of nanofluid. Int. J. Heat Mass Transf. 2015, 89, 799-808. [CrossRef]

27. Akbar, N.S.; Raza, M.; Ellahi, R. Copper oxide nanoparticles analysis with water as base fluid for peristaltic flow in permeable tube with heat transfer. Comput. Meth. Prog. Biomed. 2016, 130, 22-30. [CrossRef] [PubMed]

28. Oztop, H.F.; Abu-Nada, E. Numerical study of natural convection in partially heated rectangular enclosures filled with nanofluids. Int. J. Heat Fluid Flow 2008, 29, 1326-1336. [CrossRef]

29. Tiwari, R.K.; Das, M.K. Heat transfer argument in a two-sided lid-driven differentially heated square cavity utilizing nanofluids. Int. J. Heat Mass Transf. 2007, 50, 2002-2018. [CrossRef]

30. Kakac, S.; Pramuanjaroenkij, A. Review of convective heat transfer enhancement with nanofluids. Int. J. Heat Mass Transf. 2009, 52, 3187-3196. [CrossRef]

31. Povstenko, Y. Linear Fractional Diffusion-Wave Equation for Scientists and Engineers; Springer: Basel, Switzerland, 2015.

32. Stankovic, B. On the function of E. M. Wright. Publ. I'Inst. Math. 1970, 10, 113-124.

33. Cao, Z.; Zhao, J.; Wang, Z.; Liu, F.; Zheng, L. MHD flow and heat transfer of fractional Maxwell viscoelastic nanofluid over a moving plate. J. Mol. Liq. 2016, 222, 1121-1127. [CrossRef]

34. Pan, M.; Zheng, L.; Liu, F.; Zhang, X. Modeling heat transport in nanofluids with stagnation point flow using fractional calculus. Appl. Math. Model. 2016, 40, 8974-8984. [CrossRef]

(C) 2017 by the authors. Licensee MDPI, Basel, Switzerland. This article is an open access article distributed under the terms and conditions of the Creative Commons Attribution (CC BY) license (http:/ / creativecommons.org/licenses/by/4.0/). 